\title{
Evaluation of CCD cameras for beam profile monitoring with high intensity particle beams traversing gases
}

\author{
Raphael Hampf ${ }^{1 *}$, Andreas Ulrich ${ }^{1}$ and Jochen Wieser ${ }^{2}$
}

\author{
*Correspondence: \\ raphael.hampf@tum.de \\ 1Physik Department, Technical \\ University Munich, 85748 Garching \\ bei München, Germany \\ Full list of author information is \\ available at the end of the article
}

\begin{abstract}
Measurements of the absolute sensitivity of three different optical cameras are presented. An absolutely calibrated tungsten strip-lamp was used for calibrating the devices. An experimental method for determining the solid angle which is accepted by the combination of the cameras with a broadband apochromatic lens is described. The results with five bandpass filters between $337 \mathrm{~nm}$ and $740 \mathrm{~nm}$ are shown. The signal to noise ratio and the spatial resolution of the camera systems is also discussed.
\end{abstract}

Keywords: Optical instruments and equipment, Imaging and optical processing, Scanners, Image intensifiers, Image converters, Imaging detectors and sensors

PACS: $07.60 .-j ;$ 42.30.-d; 42.79.Ls; 42.79.PW

\section{Introduction}

CCD detectors have become powerful tools to observe 2-dimensional processes in various scientific fields. Quantitative measurements require a profound knowledge of the detector performance and the associated optical instrumentation. Absolute measurements of the sensitivity of optical detectors are often quite difficult. Here we describe the characterization of 2-dimensional optical detectors for determining absolute crosssections for optical emission from various gases excited by particle beams. A specific goal of these studies is to develop techniques for determining beam profiles of very intense ion beams. The motivation for purely optical beam profile measurements is based on the fact that alternative beam profile monitors, such as solid-state scintillators, grids, or wires would be destroyed by the beam. This can be the case when the absolute power of the beam is high, or the beam is focused to such small areas that solid materials get damaged. The optical beam profile measurement uses a gas target which is observed by appropriate camera systems. Various experimental studies have already been performed in that direction [1-7]. Here we describe first the concept of our calibration measurements followed by the results for specific cameras. The sensitivity, the signal to noise ratio and the spatial resolution are presented.

(c) The Author(s). licensee Springer on behalf of EPJ. 2020 Open Access This article is licensed under a Creative Commons Attribution 4.0 International License, which permits use, sharing, adaptation, distribution and reproduction in any medium or format, as long as you give appropriate credit to the original author(s) and the source, provide a link to the Creative Commons licence, and indicate if changes were made. The images or other third party material in this article are included in the article's Creative Commons licence, unless indicated otherwise in a credit line to the material. If material is not included in the article's Creative Commons licence and your intended use is not permitted by statutory regulation or exceeds the permitted use, you will need to obtain permission directly from the copyright holder. To view a copy of this licence, visit http://creativecommons.org/ licenses/by/4.0/. 


\section{Methods}

The sensitivity measurements of optical detectors are in general either based on absolutely calibrated light sources or the comparison with detectors with a known sensitivity. Here we used an absolutely calibrated tungsten-strip lamp (Wi17/G Osram) [8]. The central part of this lamp provides absolute values for its light emission given in $m W /\left(\mathrm{cm}^{2}\right.$. $n m \cdot s r)$. This value is the spectral radiance and will be called $L_{\lambda}$ from here on. The specified area has a size of $0.8 \mathrm{~mm} \times 2.0 \mathrm{~mm}$ and is marked by a notch on the edge of the tungsten strip. The tungsten strip is mounted in a glass housing with a quartz window. The rear side of the housing is tilted to avoid reflections. The emission of the lamp used in this experiment is specified for an electrical current of $16.61_{4} \mathrm{~A}$ and an operating voltage of $9.30{ }_{9} \mathrm{~V}(\mathrm{P} \approx 154 \mathrm{~W})$. The spectral radiance distribution is tabulated for a wavelength range from $250 \mathrm{~nm}$ to $2600 \mathrm{~nm}$ in steps of $5 \mathrm{~nm}$, with an uncertainty of $2 \%$. The lamp was operated with a stabilized dc power supply. The current was measured by picking off the voltage from a high precision $(100.00 \pm 0.01) m \Omega$ resistor. The main concept of the calibration measurement was to record two-dimensional images of the specified area of the tungsten-strip lamp. The optical setup is shown in Fig. 1. To make sure that only a calibrated surface is used for data taking we reduced the size of the observed region of interest (ROI) of the image to roughly $0.6 \mathrm{~mm} \times 1.7 \mathrm{~mm}$. Our concept was to observe the specified light source with the identical optical setup used for the beam profile measurements mentioned in the introduction. An apochromatic objective lens (JENOPTIK UV-VIS-IR $60 \mathrm{~mm}$ 1:4 APO Macro [9]) was used as an imaging optics in front of the detectors. It was always operated with an aperture setting $\mathrm{f} / 4(D / f=1 / 4)$. Specific wavelength ranges, which were of interest for the beam profile measurements, were selected with optical bandpass filters (Edmond Optics, traditional coated filters with center wavelength (CWL) $390 \mathrm{~nm}$ and $740 \mathrm{~nm}$, as well as hard coated filters with CWL 337nm, 473nm, and 589nm). The FWHM of the bandpass filters was typically 10nm (Fig. 6). Since very sensitive cameras were used in combination with the imaging optics, the light of the calibrated lamp had to be strongly attenuated for recording the images. Several neutral density filters were placed in front of the light source for that purpose [10]. The combined attenuation was

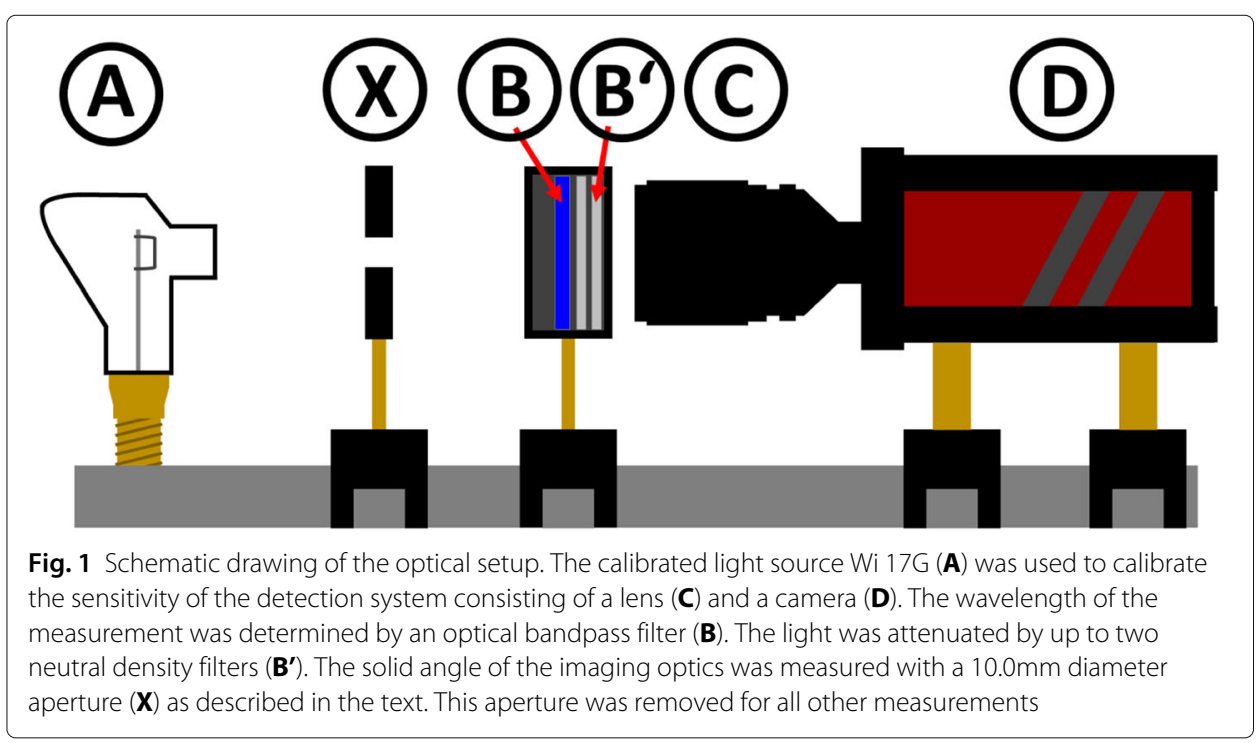


chosen between $10^{4}$ and $10^{7}$ depending on the bandpass filters used and the sensitivity of the cameras in the respective wavelength region. The transmission spectrum of the neutral density filters was taken from the data sheet. It should be noted that the transmission is not necessarily constant in the designated wavelength range from $250 \mathrm{~nm}$ up to $2000 \mathrm{~nm}$. While the transmission of the filters with nominal OD 2 and OD 1 did not deviate more than $25 \%$ from the nominal value, for the filters for OD 3 and OD 4 it deviated up to a factor of 3 in the UV and IR wavelength region. It is important to note that the filters had to be tilted slightly with respect to each other to avoid the influence of multiple reflections as shown in Fig. 2.

Three different types of cameras were used. The first one is a pure CCD camera (ATIK $383 \mathrm{~L}+$ ). The second one is an iCCD or intensified CCD camera (PI-Max4 1024f). The primary photo-detector of this camera is a vacuum device with an S20 photocathode, producing photoelectrons, which are further on accelerated and multiplied in a mircrochannelplate (MCP) amplifier. The (intensified) output of the MCP leads to scintillation on a scintillator which finally is observed with a CCD detector. The third camera is an EMCCD camera. Here the electrons that are generated in the pixels are amplified from pixel to pixel during read-out. The camera was mounted at a distance of about $40 \mathrm{~cm}$ to the tungsten-strip lamp. The solid angle that is captured by the lens was determined in the following way: A circular aperture with $10.0 \mathrm{~mm}$ diameter, which is smaller than the aperture of the lens, was placed between the lamp and the lens. This aperture was moved with respect to the lamp and the camera. At close distances to the lamp, the accepted solid angle is defined by the aperture of the lens. At a certain distance the introduced aperture cuts in, and reduces the light intensity by a $1 / x^{2}$ law. The solid angle accepted by the lens and the camera was determined by the position at which the aperture started to

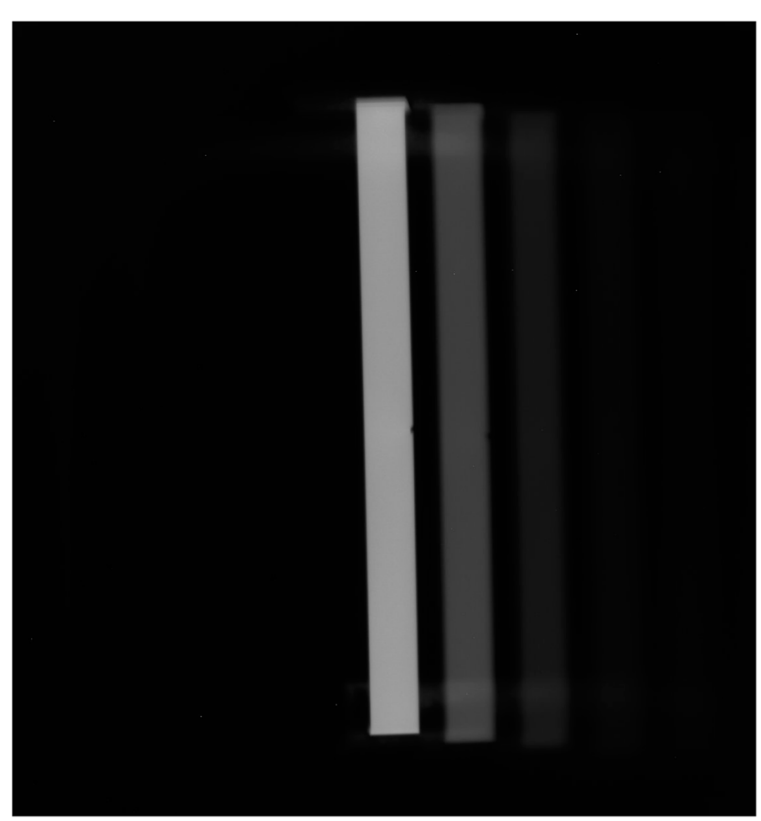

Fig. 2 An image of the calibrated tungsten-strip of the calibration lamp is shown. The weaker signal to the right side, are reflections of that tungsten-strip on slightly tilted neutral density filters. This tilt was deliberately introduced to avoid that these reflections overlay with the original image 
reduce the recorded light intensity. Due to the finite thickness of the introduced aperture, we have a smooth transition from constant light intensity towards decreasing behavior.

The experiment was set up on an optical bench with scales for the position. The dimension of all parts used were measured, enabling the determination of the exact position of all components. The results are shown in Fig. 3 exemplary for one camera system. The solid angle can be calculated using the area of the aperture and the distance to the light source. It was determined to $(3.84 \pm 0.28) \cdot 10^{-3} \mathrm{sr}$ for camera $1,(2.85 \pm 0.15) \cdot 10^{-3} \mathrm{sr}$ for camera 2 and $(2.88 \pm 0.18) \cdot 10^{-3} \mathrm{sr}$ for camera 3 . The mounts of the cameras were placed in the same position for all three cameras. Due to the different dimensions of camera 1 , the focusing was changed accordingly, leading to a different solid angle compared to the other two cameras. The errors can be calculated from the uncertainty of the diameter of the aperture (precision $0.1 \mathrm{~mm}$ ), and the position of the kink where the intensity starts to drop (precision $5 \mathrm{~mm}$, see Fig. 3). It has to be noted that a calculation of the solid angle based on the nominal aperture of the lens cannot easily be used for a thick objective lens, because the exact position of the aperture and the optical path of the light is not well known for the user.

\section{Results and discussion}

We tested three cameras with monochrome CCD detectors. These cameras were also used for the beam profile measurement experiments, which will be presented elsewhere. Type and parameters of the cameras are listed in Table 1.

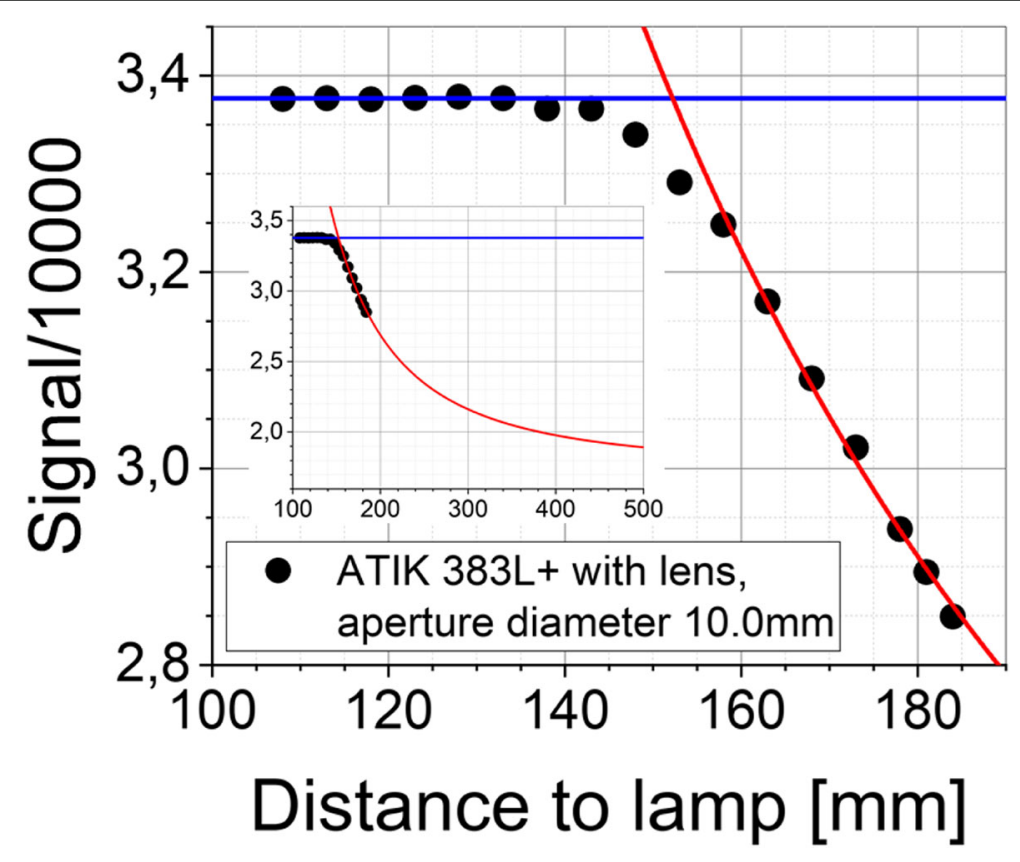

Fig. 3 The light intensity recorded by the camera (Signal) is shown versus the distance of the aperture $(\mathbf{X}$, in Fig. 1) to the tungsten-strip. The concept of the measurement is described in the text. The constant intensity value for distances shorter than $143 \mathrm{~mm}$ corresponds to a solid angle of $3.84 \cdot 10^{-3} \mathrm{sr}$. In red the $I(x)=I_{0} \cdot x_{0}^{2} / x^{2}+U$ fit function is plotted. The blue line is the constant level of intensity before the point of reduction. The smaller plot inside the main plot shows a wider distance and signal range in the way it is expected to evolve 
Table 1 Information taken from [11-13]

\begin{tabular}{lllll}
\hline No. & Name & Manufacturer & Type & Chip \\
\hline 1 & ATIK 383L+ & Atik Camera & CCD & KAF-8300 \\
2 & PI-Max4 1024f & Princeton Instruments & iCCD & e2v CCD 47-10 \\
3 & ProEm+ 512B & Princeton Instruments & EMCCD & e2v CCD 97B \\
\hline
\end{tabular}

All cameras show a linear behavior with respect to the exposure time for all bandpass filters. An example is shown in Fig. 4. The dynamic range of the cameras was only used up to roughly $60 \%$ of its full range to avoid saturation effects (40000 ADU out of 65535 ADU, where ADU, or Analog-Digital-Unit, is the signal value converted into a digital output number by the Analog-to-Digital Converter or ADC). For all cameras and spectral regions tested we used the images that were recorded with our setup as described above. Figure 5 shows an example of the raw data that were recorded, which is an image of the specified region of the tungsten-strip lamp, recorded through the corresponding bandpass and neutral density filters. The sensitivity of the cameras in the specific wavelength ranges were determined in the following way.

The ROI on the tungsten-strip lamp was used as the absolutely calibrated light source. The value, which is tabulated for that light source, provides the light intensity per unit area, band width, and solid angle (in this case in the units $m W /\left(\mathrm{cm}^{2} \cdot n m \cdot s r\right)$ ). The relevant area on the detector is given by the area of the ROI and the reproduction scale between the camera and the tungsten-strip (see yellow box in Fig. 5). The reproduction scale was determined by imaging objects with known dimensions (here a scale paper) at the same distance from the camera (i. e. the image sensor) as the tungsten strip. Furthermore, the tungsten strip itself is defined with a $1.6 \mathrm{~mm}$ width, and hence can be used as a scale. The

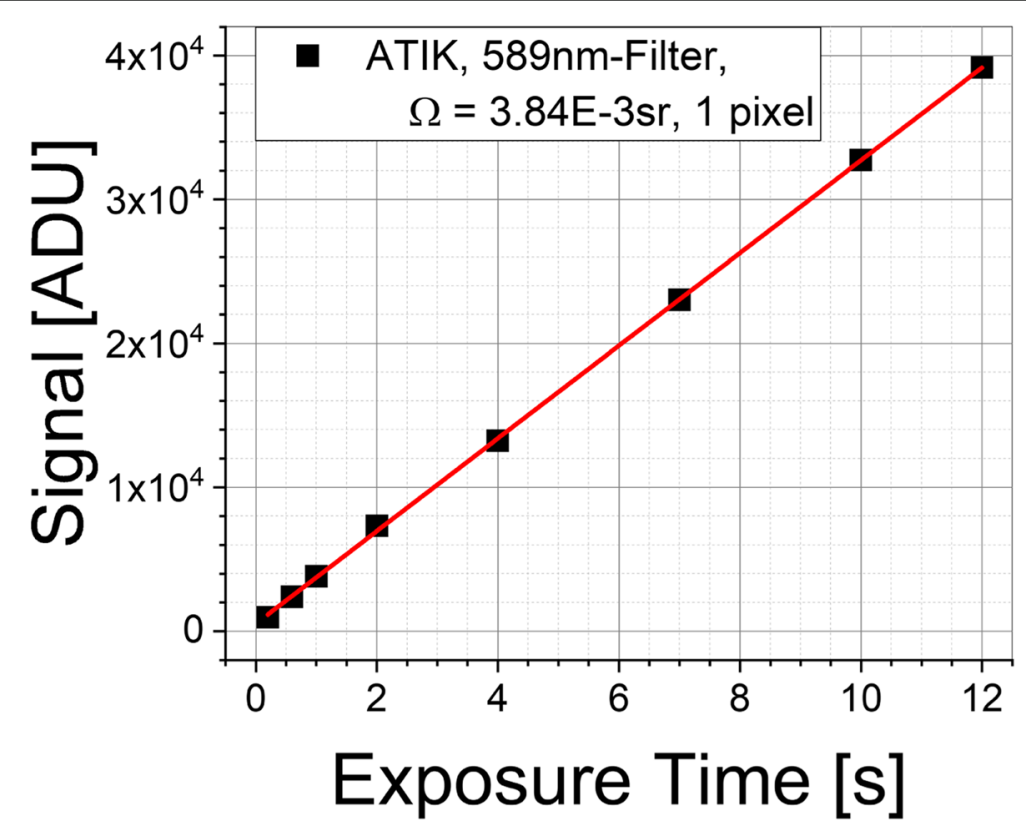

Fig. 4 The grey value (in Bits) is shown versus the exposure time as an example using the bandpass filter with a center wavelength (CWL) of $589 \mathrm{~nm}$. It shows the linear behavior of the camera with respect to exposure time. All three cameras show the same linear behavior with all bandpass filters used 


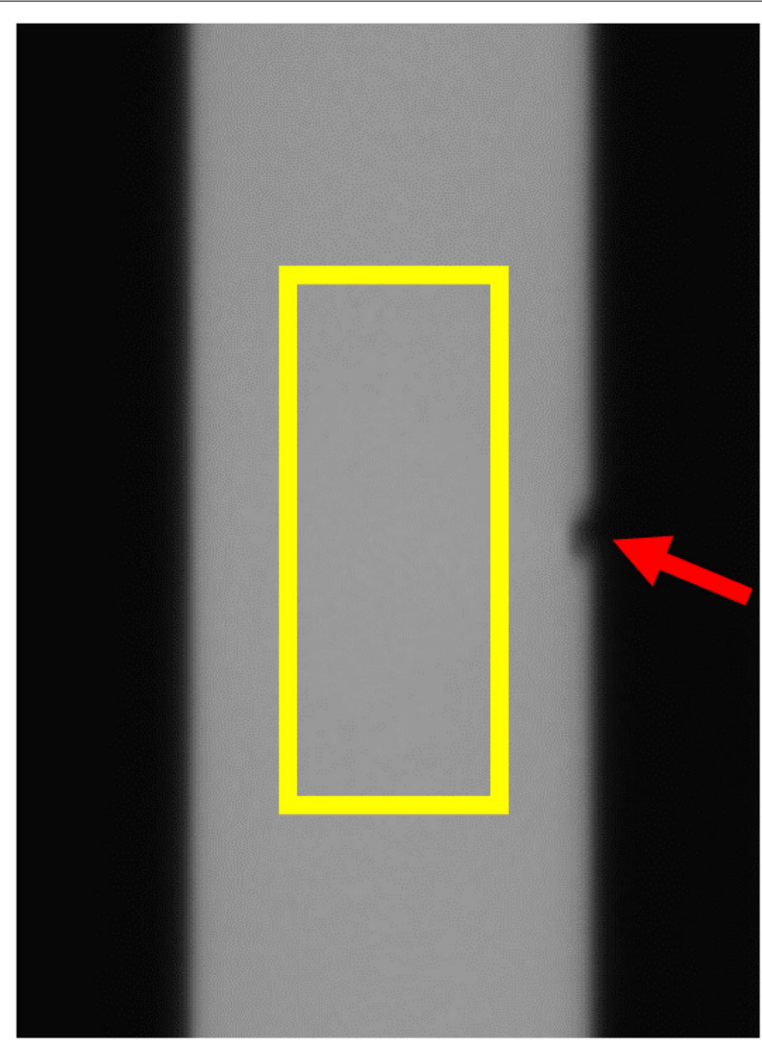

Fig. 5 An example of the raw data is shown. It is an image of the tungsten-strip of the calibration lamp. The calibrated region is schematically shown with the rectangular box. The position of the calibrated region can be found using the notch (arrow)

bandwidth is taken into account by multiplying the interpolated emission curve of the lamp with the transmission curve of the bandpass filter (see Fig. 6).

The solid angle for the different cameras was determined as described above. As a last step to determine the calibrated light, reaching the detector, the transmission through the neutral density filters and the transmission of the lens was taken into account, using manufacturer supplied data. At the end the power of the light can be converted into a photon flux by dividing by the energy of the photons. This process thus can be summarized by the following equation: The spectral radiance of the standard light source was weighted by the (wavelength dependent) transmission functions of the applied color- and neutral-density filters (given by the manufacturer), and integrated over the wavelength, the fiducial area of the lamp, and the accepted solid angle of the lens. The result, the photons incidenting on one pixel, $N_{p h \text {,pixel }}$, was normalized to the applied magnification scale and pixel size, as well as the photon energy:

$$
N_{\text {ph,pixel }}=\frac{\Delta t}{E_{P h}} \frac{M a g^{2} \cdot l_{\text {pix }}^{2}}{A_{\text {fid }}} \int_{\Omega_{\text {lens }}} \int_{B P F} \int_{A_{f i d}}\left(L_{\lambda} \cdot T(\lambda)\right) d A d \lambda d \Omega,
$$

where $\Delta t$ is the exposure time, $E_{p h}=h c / \lambda$ the mean photon energy of the photons passing the bandpass filter, $l_{p i x}$ the pixel width of the detector (given by the manufacturer), Mag the linear magnification scale, $L_{\lambda}$ the spectral radiance, $T_{\lambda}$ the total transmission 


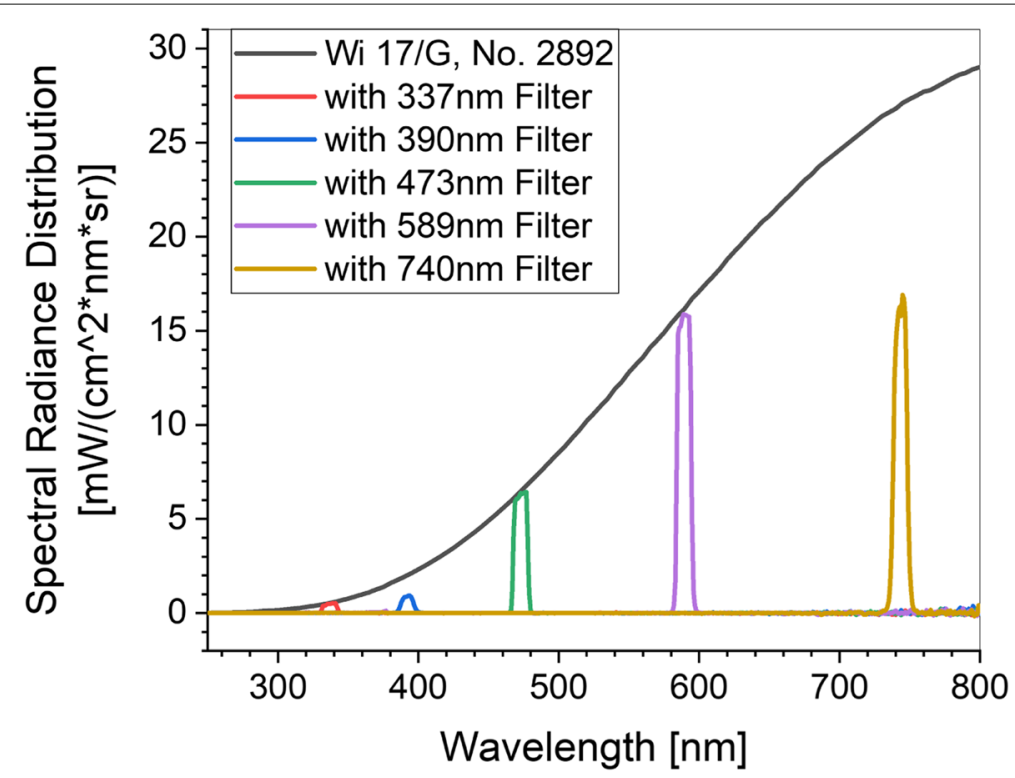

Fig. 6 The spectral distribution of the light which is used for the calibration of the detector systems is shown. The black curve shows the emission of the calibration lamp versus the wavelengths. The individual peaks show the multiplication of the curves with the filter transmission

through the various optical components, and $\Omega_{\text {lens }}$ the solid angle accepted by the lens. The spectral radiance is given by

$$
L_{\lambda}=\frac{d^{3} \Phi}{d A d \Omega d \lambda}
$$

and the total transmission $T(\lambda)$ is the product of the transmission of the respective neutral density filters used $T_{N D}(\lambda)$, the bandpass filter $T_{B P F}(\lambda)$, and of the objective lens $T_{\text {lens }}(\lambda)$

$$
T(\lambda)=T_{N D}(\lambda) \cdot T_{B P F}(\lambda) \cdot T_{\text {lens }}(\lambda) .
$$

As mentioned above the spectral radiance is constant over the specified area on the strip of the lamp. The same applies for the solid angle, particularly using an apochromatic lens. This simplifies Eq. (1) to

$$
N_{\text {ph,pixel }}=\frac{\Delta t}{E_{P h}} M a g^{2} \cdot l_{\text {pix }}^{2} \cdot \Omega_{\text {lens }} \int_{B P F} L_{\lambda} \cdot T(\lambda) d \lambda .
$$

In practise $L_{\lambda} \cdot T(\lambda)$ is calculated by interpolating the curve of the spectral radiance in a way that the radiance $L(\lambda)$ at a certain wavelength can be multiplied with the corresponding transmission $T(\lambda)$.

The signal which is created by this calibrated light on the detector is determined by taking the average of the grey value that is recorded by the camera in the ROI. This provides the grey value for one pixel of the camera for the absolute flux of light reaching its surface. We assume that all pixels of the camera have the same sensitivity, since at least in the ROI a normal (Gaussian) distribution of the pixel intensity could be observed. The width of the Gaussian represents both the photoelectron-statistics in a potential well (essentially photon statistics times conversion efficiency per pixel - shot noise) and readout noise, together with the amplification, i.e. the conversion factor of "measured electrons" into ADU. Note that ADU is not the "physical unit" governing statistics. For example for camera 1 , at an exposure in a way that 40000 ADUs were reached in the ROI, the width of the 
Gaussian was 780 ADU (respectively 330 ADU StdDev, instead of the "expected" standard deviation of 200 ADUs for 40kADU total). Note, there are less photo-electrons in a pixel than displayed ADUs, thus the "shot-noise times gain" is larger than the Sqrt(ADU)! In this case an "amplification" or gain factor of 2.7 ADU for every electron in a pixel's potential well was calculated for the ATIK-camera $\left((330 / 200)^{2}=2.7\right)$. Furthermore, the nominal gain factor can be calculated by another approach, using data sheet values of the sensor (KAF-8300 [14]): The saturation is reached at $25500 \mathrm{e} / \mathrm{pixel}$. This corresponds to the maximum signal value in ADU of 16bit $=2^{16}=65536$. Hereby, a gain factor of 2.6 ADU/e can be derived. Atik itself publishes a value of 0.41 electrons/ADU, respectively a gain of 2.4 ADU/electron [15]. This is close to, but not exactly the factor of 2.7 ADU/e, calculated from noise, respectively full well capacity considerations.

The signal which is relevant for the calibration was calculated by subtracting the background from the grey value. The background was measured by switching off the calibration lamp and taking the average value in the same ROI.

The goal of the measurement is to provide the sensitivity of the camera systems per incident photon for wavelength positions measured. To obtain these values, we took the number of pixels in the ROI, multiplied it with the average signal per pixel and compared it with the number of incoming photons hitting the image of the ROI on the detector surface. Figure 7 shows the sensitivity in ADU per photons for each of the three camera systems. The result is shown on a logarithmic scale for comparing all three camera systems with respect to the wavelength in Fig. 8. The rather large error bars have its origin mainly in the uncertainty of the spectral transmission of the several filters used, especially the high-absorbtive neutral density filters. As a first result, it can be seen that the sensitivity of the camera with the image intensifier (PI-Max4) is more sensitive in the blue and violet wavelength region than the purely silicon based cameras, an important aspect when matching the right camera for a selected optical transition. This performance was expected, though, since the photocathode of the image intensifier of the PI-Max4 is a multialkali photocathode, which is generally more sensitive towards the blue/violet wavelength region. Please note that this is a calibration of light intensity directly to ADU, a parameter usually needed when performing quantitative measurements with a digital camera. We do not determine the quantum efficiency of the camera's detector. However, the QE can be derived by dividing the digital ADU by the camera's gain. For e.g. the ATIK camera, using the provided gain factor of 2.4, the QE can be calculated from the ADU-sensitivity values, reaching about $45 \%$ efficiency at $450 \mathrm{~nm}$. This coincides well with published values for pn-CCD detectors [16], however deviates in details from published values.

Besides the absolute sensitivity it is important to consider also the signal to noise ratio (SNR) of the camera systems for different operating conditions. The signal was determined as described above, while the noise was derived as follows: First, two images with identical settings were subtracted from each other. A histogram of the difference values in the ROI was plotted, the standard deviation from the mean value was divided by the square root of 2 (having the sum-noise of two pictures!) and the result was taken as the noise. As "noise" we subsume all fluctuations in the output, being it thermal noise, readout-noise, or photo-electron statistics within each pixel (shot noise). For high signal"count", photoelectron statistics will dominate, while for very long exposure times and low signal count thermal noise might play a role, in spite of the cooled detector. For very low 


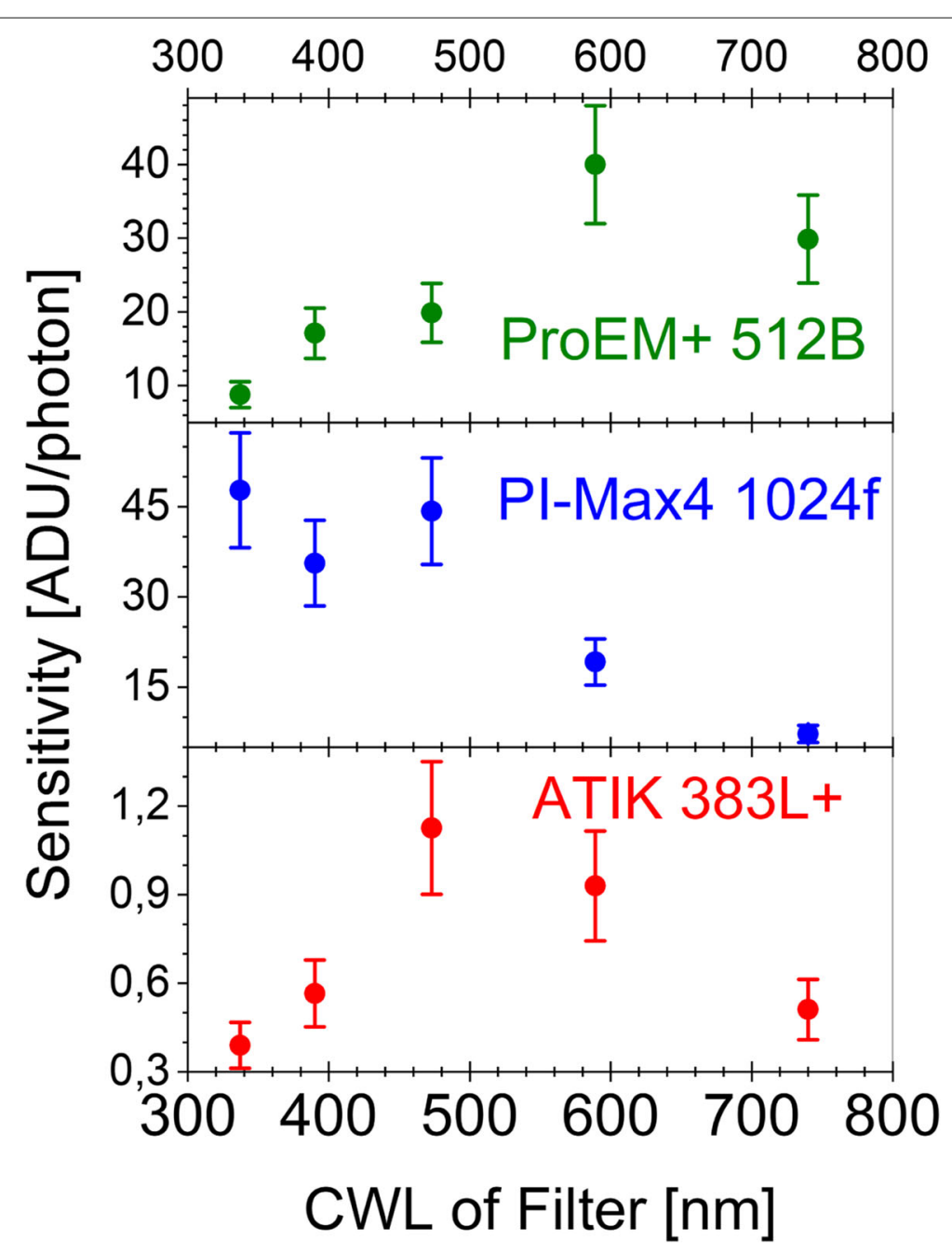

Fig. 7 The sensitivity of the three camera systems which have been tested is shown versus the center wavelength (CWL) of the filters used to determine the wavelength position of the measurement. Due to the S20 cathode of the image intensifier the PI-Max4 has a high sensitivity in the blue and UV wavelength region

signal count, readout-noise always is an issue. The signal is varied by varying the exposure time for a fixed flux of light from the calibration lamp. The result is shown in Fig. 9.

It is interesting to see that the intensified cameras have a lower SNR for a given signal value. On the other hand, a certain signal is obtained with a much shorter exposure time in the case of the intensified cameras. These data may help to choose the right detector for given experimental boundary conditions.

Figure 10 shows the SNR for the three cameras for the 5 different bandpass filters in dependence of the exposure of a single pixel on the camera chip. As exposure we define the number of photons reaching the camera's detector in the ROI. The exposure is then normalized to the number of pixels in the ROI. This is 2400 pixels for the ATIK 383L+, 396 pixels for the PI-Max4 1024f and 270 pixels for the ProEM+ 512B. Better SNR can be obtained with the silicon based detector without intensifier (ATIK) but at the expense of a much longer exposure time (note the logarithmic scale of the exposure and that the pixel size is much smaller). With hardware binning this exposure time could potentially 


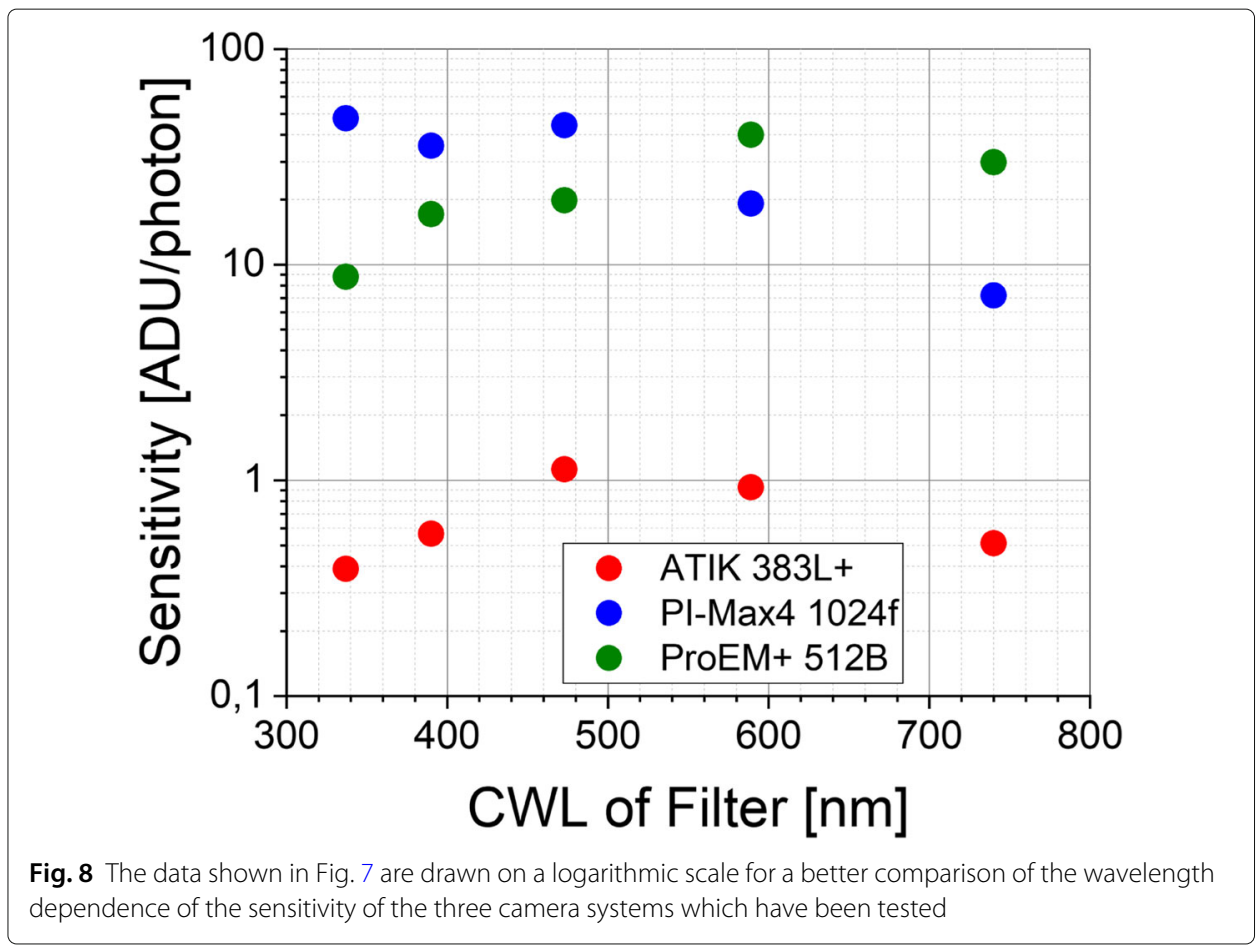

be reduced. The minimum exposure of the ATIK $383 \mathrm{~L}+$ was limited by the mechanical shutter $\left(\tau_{\min }=0.2 s\right)$.

Additionally to sensitivity, linearity, and signal-to-noise ratio, the "spatial resolution" is a critical component of an optical system. Here, simulating a "sharp beam profile", we measured the "line-spread-function", observing the sharp cutoff of intensity at one side

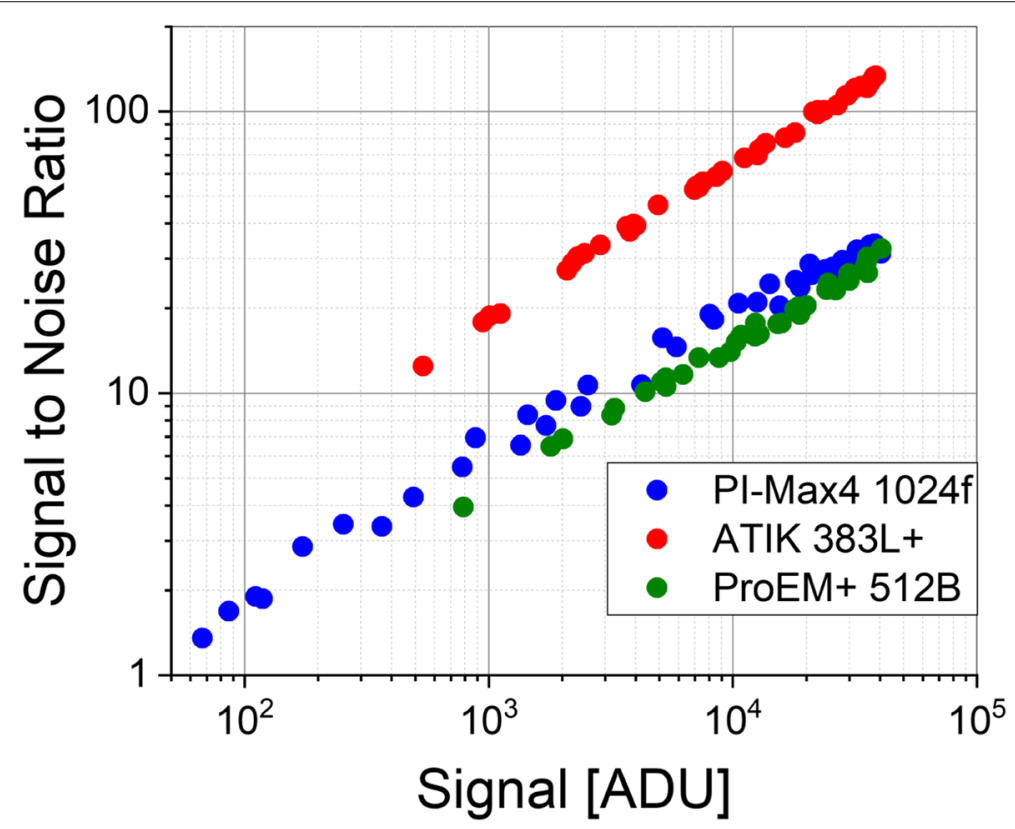

Fig. 9 The signal to noise ratio (SNR) of the three camera systems is shown versus the absolute signal. The different signal strengths were obtained by varying the exposure time recording the light source with fixed intensity 


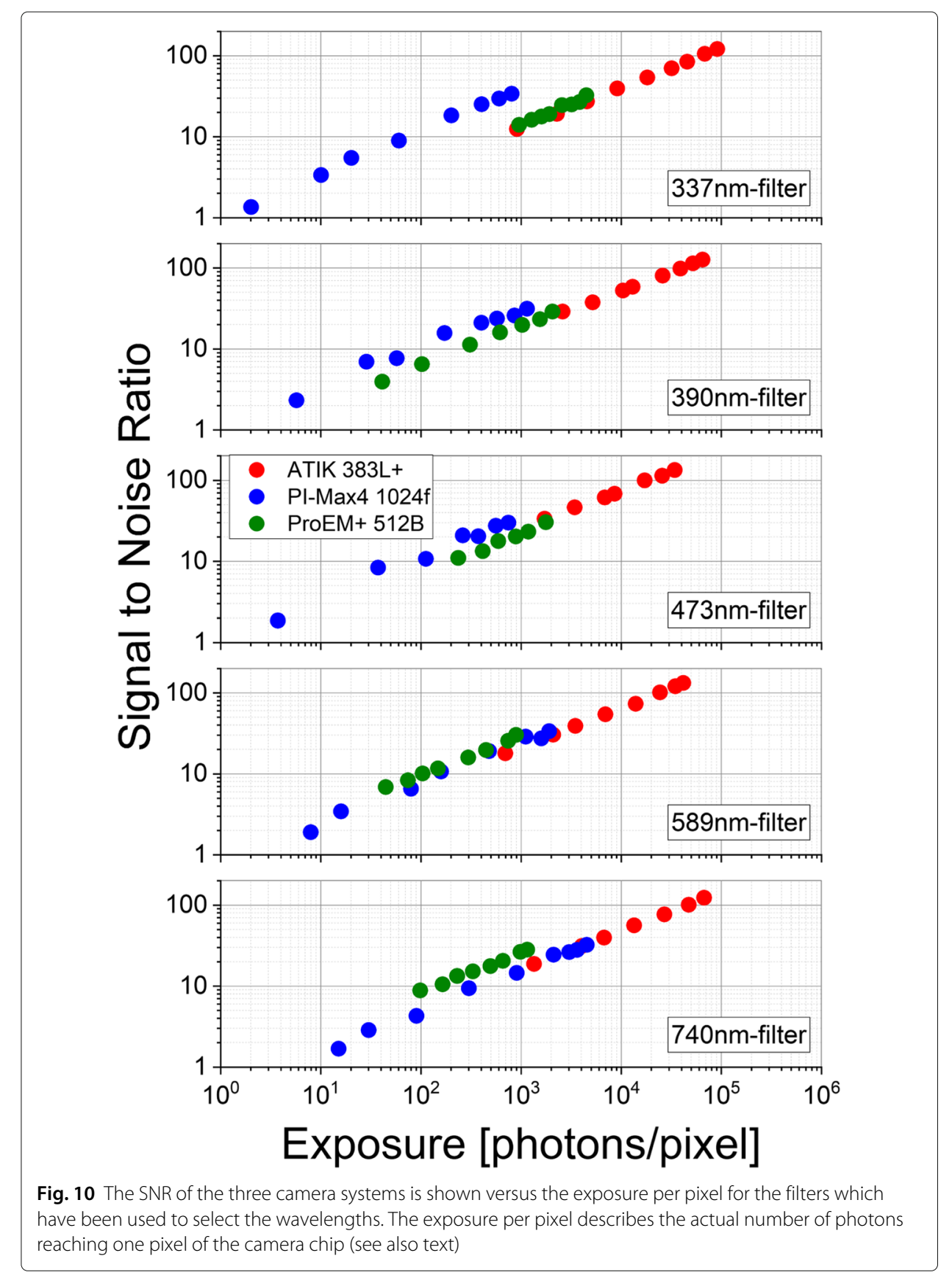

of a homogeneously backside illuminated precision slit (see Fig. 11). In an ideal system the data would show a step-function. Any deviation from this step function is resolution decreasing. Here, the following indication of "resolution" is given: the pixel-distance for the $10 \%$ to $90 \%$ intensity value can be, and often is, assumed to be "the resolution"; this is shown in (Fig. 12) exemplarily for the ATIK 383L+ camera. The results are shown in Table 2, indicating a 10-90\% resolution always worse than about 3 pixel.

Please note that the magnification was for all cameras essentially 1:4 (image-size : object-size), while the pixel sizes differ significantly, being $5.4 \mu \mathrm{m} \times 5.4 \mu \mathrm{m}$ for the ATIK-, $13 \mu \mathrm{m} \times 13 \mu \mathrm{m}$ for the PI-Max4-, and $16 \mu \mathrm{m} \times 16 \mu \mathrm{m}$ for the ProEm-camera, respectively. 


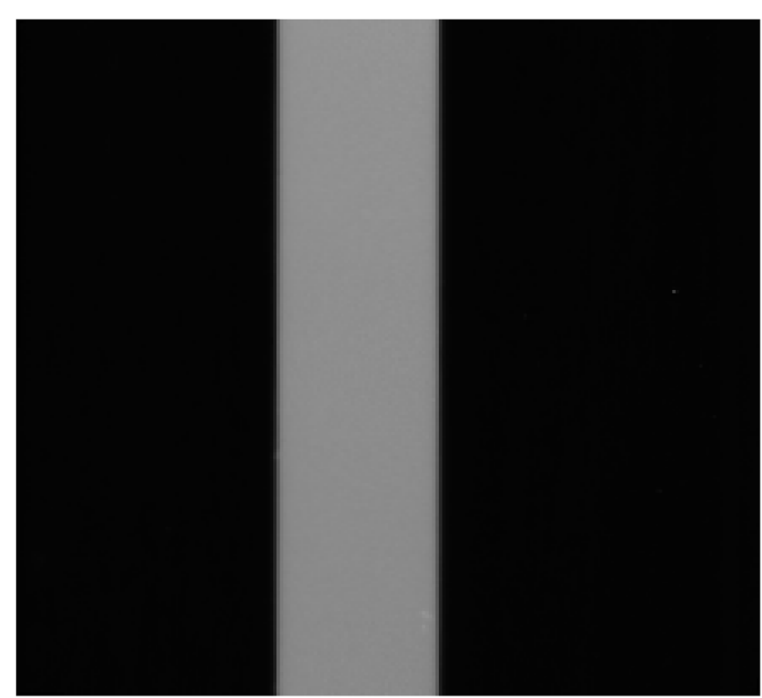

Fig. 11 Backside illuminated slit for measuring the spatial resolution of the camera systems used

The resolution hence is here not limited by the lens: Particularly using the $10-90 \%$ conditions, the large-pixel-size cameras should sport a significantly steeper step-function compared to the small pixel ATIK. No thorough investigation was performed what exactly caused the deviation from a step-function; "blooming" into the next pixel may be the most likely explanation, while micro-vibrations of the entire setup during illumination cannot be excluded as well. It should be noted, however, that this line-spread results in an "objectresolution" of about $60 \mu \mathrm{m}$ (ATIK), respectively $170-220 \mu \mathrm{m}$ for the intensified cameras (PI-Max, ProEM) (spatial resolution in pixel x pixel-size x 1 /magnification). For a "real" beam profile measurement setup, hence not only the quality of the imaging optics, but

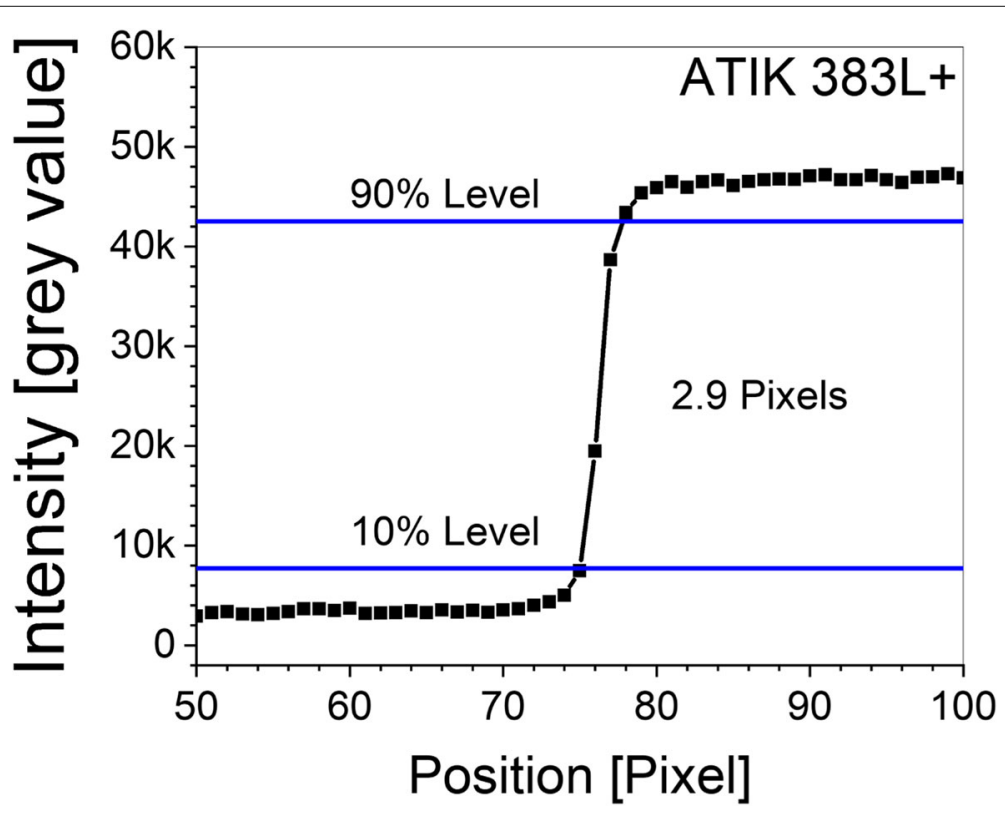

Fig. 12 Exemplary demonstration of the 10\% to $90 \%$ method for determining the spatial resolution of the camera system. The curve shows the intensity profile around the edge of the slit 
Table 2 Information taken from [11-13]

\begin{tabular}{lllllll}
\hline No. & Name & Pixel size & Active pixels & $\begin{array}{l}\text { Gain } \\
\text { setting }\end{array}$ & $\begin{array}{l}\text { Spatial } \\
\text { Resolution } \\
\text { [pixels] }\end{array}$ & Magnification \\
\hline 1 & ATIK & $5.4 \mu \mathrm{m} \times \mathbf{\mu m} \times 5.4 \mu \mathrm{m}$ & $3326 \times 2504$ & 1 & $2.9 \pm 0.2$ & $1: 3.9$ \\
2 & PI-Max4 & $13 \mu \mathrm{m} \times 13 \boldsymbol{\mu m}$ & $1024 \times 1024$ & 90 & $4.5 \pm 0.5$ & $1: 3.7$ \\
3 & ProEm+ & $16 \mu \mathrm{m} \times 16 \mu \mathrm{m}$ & $512 \times 512$ & 100 & $2.7 \pm 0.2$ & $1: 4.0$ \\
\hline
\end{tabular}

also the pixel size of the detector (as well as the magnification of the setup) needs to be taken into account, since a typically 3 pixel resolution (10-90\% value) can realistically not be undercut by much.

\section{Conclusions}

This work summarizes and presents techniques, which we have developed in the context of optical beam diagnostic experiments for intense particle beams. We see the strength of this work in developing a technique to determine the absolute sensitivity of camera systems over a wide wavelength range. While quantum efficiencies and gain factors often are given in the datasheet of the respective cameras, the measured values deviate up to $30 \%$ from the calculated efficiency values. Setting up an experiment, the published data are sufficient; for precise measurements, a dedicated calibration is recommended. In the context of our work this is important for the determination of emission cross-sections for particle beam induced light emission processes. This implies also to know the solid angle for the emission measurements as precisely as possible, particularly, using a "thick lens". Here, we also describe a solution for this problem. Measurements for determining the spatial resolution of camera systems are presented as well.

Exemplary measurements of beam profile monitoring have been performed at the Munich Tandem van de Graaff accelerator with a heavy ion beam of $1 \mathrm{~mm}$ diameter traversing argon and neon. The parameters of all cameras were sufficient in these experiments with respect to sensitivity and spatial resolution. A systematic study of the issues of beam profile measurements using the three types of camera systems described here, will be published in a forthcoming paper.

Acknowledgements

The authors acknowledge the financial support by the Federal Ministry of Education and Research of Germany in the framework of the APPA R\&D project 05P2015 and the Maier-Leibnitz-Laboratory. The authors acknowledge the collaboration and discussion with the Accelerator Beam Diagnostics Department at GSI Darmstadt, Peter Forck and Serban Udrea, who also provided the ProEM+512B camera for the measurements.

\section{Authors' contributions}

The concept for this work was provided by AU. The experiments were performed by RH supported by JW and AU. Data analysis was done by RH supported by JW and in discussion with AU. Discussion, interpretation and the text for the manuscript were provided by all three authors. The author(s) read and approved the final manuscript.

\section{Funding}

The authors acknowledge the financial support by the Federal Ministry of Education and Research of Germany in the framework of the APPA R\&D project 05P2015 and the Maier-Leibnitz-Laboratory and the funding by the Hochschulprogramm der GSI (GSI Helmholtzzentrum für Schwerionenforschung) - TMULRI1719. Open Access funding enabled and organized by Projekt DEAL.

Availability of data and materials

The datasets used and/or analysed during the current study are available from the corresponding author on reasonable request. 


\section{Author details}

${ }^{1}$ Physik Department, Technical University Munich, 85748 Garching bei München, Germany. ${ }^{2}$ Excitech GmbH, 26419

Schortens, Germany.

Received: 24 September 2019 Accepted: 29 November 2020

Published online: 19 December 2020

\section{References}

1. Varentsov D, et al. (2008) Transverse Optical Diagnostics for Intense Focused Heavy lon Beams. Contrib Plasma Phys 48:586

2. Forck P (2010) Minimal invasive beam profile monitors for high intense hadron beams. In: Conf. Proc. IPAC. JACOW Publishing, Kyoto. p 1261

3. Becker F, et al. (2012) Beam induced fluorescence - profile monitoring for targets and transport. In: Conf. Proc. of HB. JACOW Publishing, Beijing. p 798

4. Andre C, et al. (2014) Optimization of beam induced fluorescence monitors for profile measurements of high current heavy ion beams at GSI. In: Conf. Proc. IBIC. JACOW Publishing, Monterey. p 412

5. Udrea S, et al. (2017) Development of a fluorescence based gas sheet profile monitor for use with electron lenses: optical system design and preparatory experiments. In: Conf. Proc. IBIC. JACOW Publishing, Grand Rapids. p 359

6. Salehilashkajani A, et al (2019) Commissioning of the prototype for a new gas curtain beam profile monitor using beam induced fluorescence for HL-LHC. In: Conf. Proc. IPAC. JACOW Publishing, Geneva

7. HD Zhang, et al (2019) Development of supersonic gas-sheet-based beam profile monitors. In: Conf. Proc. IPAC. JACOW Publishing, Geneva

8. OSRAM GmbH (1987) Prüfschein Nr 2892 Normallampe Wi 17G. OSRAM GmbH, München

9. JENOPTIK Optical Systems, Data Sheet, UV-VIS-IR 60mm 1:4 APO Macro High performance lens for forensics, science and fine art, JENOPTIK Optical Systems, LLC

10. LOT-QuantumDesign GmbH, Spektrale Dichte Filter Satz, Article number: 130FA46-50, LOT-QuantumDesign GmbH, Darmstadt, Germany

11. ATIK cameras (2015) Atik 383L+ User Manual. ATIK cameras, Norwich

12. Princeton Instruments (2018) PI-Max4 Camera System Issue 9. Princeton Instruments, Trenton

13. Princeton Instruments (2012) ProEM+ System Manual, Version 1.A. Princeton Instruments, Trenton

14. ON Semiconductor (2017) KAF-8300, 3326 (H) x 2504, (V) Full Frame CCD Image Sensor, - Rev.3. ON Semiconductor, Aurora

15. Atik cameras specification tables. https://www.atik-cameras.com/wp-content/uploads/2017/02/Atik-SpecificationsTables-Feb-17.pdf. Accessed 06 Mar 2020

16. Hartmann R, et al. (2000) The quantum efficiency of pn-detectors from the near infrared to the soft X-ray region. Nucl Inst Methods Phys Res A 439:216

\section{Publisher's Note}

Springer Nature remains neutral with regard to jurisdictional claims in published maps and institutional affiliations.

\section{Submit your manuscript to a SpringerOpen ${ }^{\circ}$ journal and benefit from:}

- Convenient online submission

Rigorous peer review

- Open access: articles freely available online

- High visibility within the field

- Retaining the copyright to your article

Submit your next manuscript at $\gg$ springeropen.com 